\title{
Sex hormone-binding globulin concentrations in women with severe premenstrual syndrome
}

\author{
Maureen E. Dalton \\ M.B. B.S. \\ Dept of Endocrinology, Royal Free Hospital, London
}

\begin{abstract}
Summary
Sex hormone binding globulin (SHBG) binding capacity was measured in $\mathbf{5 0}$ women with severe premenstrual syndrome and 50 age-matched controls. The binding capacity was signicantly lower $(P=0.001)$ in the patients. This finding suggests that the levels of SHBG binding capacity could be useful in the diagnosis and may help to explain the aetiology of the premenstrual syndrome.
\end{abstract}

\section{Introduction}

Severe premenstrual syndrome (PMS) may have serious consequences including hospital admissions for asthma, epilepsy and suicide, and criminal offences ranging from manslaughter and baby battering to shoplifting and assault.

It has been accepted that total hormone concentrations may not be so biologically significant as those of the unbound fraction. Sex hormone binding globulin (SHBG) binds oestradiol and testosterone. Backström and Cartensen (1974) found a rise in total oestradiol levels in PMS. Others have suggested that oestrogen : progesterone ratio is the critical factor (Dalton, 1977) and recent work has shown that women with PMS have low levels of progesterone (Munday, Brush and Taylor, 1977). This survey was conducted to determine whether there was a change in SHBG binding capacity, resulting in changes in total oestradiol concentration, in women with severe PMS.

\section{Materials and method}

The 50 patients were recruited from a PMS clinic. Their ages ranged from 16 to 52 years and they had received no medication during the 2 months before investigations. They included 8 who had been admitted to mental hospital for premenstrual depression; 5 had made premenstrual suicide attempts, 5 had committed criminal offences premenstrually, 5 had a history of premenstrual violence, one was a premenstrual baby batterer, one a premenstrual epileptic, one a premenstrual

Address for reprints: Dept of Obstetrics and Gynaecology, The London Hospital, Whitechapel, E.1. alcoholic and one a premenstrual asthmatic. $\stackrel{\circ}{\vec{C}}$ Hirsute women and those with known thyroid and 3 . liver disease were excluded from the study. Premenstrual syndrome was diagnosed by charting the symptoms in order to check that they were recurring. in the same phase of each menstrual cycle with aI minimum of 7 consecutive days completely free from symptoms postmenstrually (Dalton, 1977).

Fifty healthy women volunteered as controls. $\overrightarrow{C D}$ They were matched for age and did not admit too having any premenstrual symptoms. They were tested in the same phase of the menstrual cycle.

Blood was usually taken on day 21 of the cycle $-\Phi$ but owing to the long distances travelled by patients, who came from all over the British Istes, 10 patients had their blood taken on the day of the clinic visit. Blood was immediately centrifuged, separated and stored at $-20^{\circ} \mathrm{C}$ until assayed for SHBG binding capacity in batches of 20 , using theo 2-tier column method of Iqbal and Johnson (1977).

The results were expressed as nmol dihydro- $\propto$ testosterone (DHT) bound per litre. There is as yet no satisfactory direct assay of SHBG.

\section{Results}

The values of SHBG binding capacity are shown in the Table. The women with severe PMS hadsignificantly lower SHBG binding capacity than the? age-matched controls, using Student's ' $t$ ' test. The' results of the PMS women were in the range seen in women with hirsutism, although none was hirsute.

No women with PMS had an SHBG binding? capacity within the normal range of this assayo $(50-80 \mathrm{nmol}$ DHT bound/l) and there was no overlap with controls.

\section{Discussion}

Many women are mis-diagnosed as having PMS because no attempt is made to chart the symptomso before diagnosis. A simple blood test which could help in making the diagnosis would therefore be ${ }^{\text {? }}$ valuable. This survey suggests that SHBG binding capacity could be a potentially useful diagnostic aid $\stackrel{\overrightarrow{\mathrm{D}}}{\mathrm{D}}$ 
TABLE 1.

\begin{tabular}{|c|c|c|c|c|}
\hline & $n$. & $\begin{array}{c}\text { nmol } \\
\text { dihydrotestosterone } \\
\text { bound } / 1\end{array}$ & s.d. & $P$ \\
\hline Premenstrual syndrome & 50 & $31 \cdot 14$ & $11 \cdot 85$ & 0.001 \\
\hline Age-matched controls & 50 & $61 \cdot 66$ & 11.94 & \\
\hline
\end{tabular}

Backström et al. (1976) found no difference from normal in SHBG binding capacity in 15 women assessed by a psychiatrist and gynaecologist as suffering from PMS with anxiety as their main symptom. The patients in the present study may have a more severe form of PMS than those in the study of Backström et al. but, because of the difficulty in grading the severity of widely differing symptoms, no attempt has been made to correlate severity with SHBG binding capacity.

The low SHBG binding capacity may reflect an alteration in total testosterone or oestradiol concentrations but preliminary studies have shown no difference in either of those hormones in these PMS women (testosterone $0 \cdot 3-1 \cdot 7 \mathrm{nmol} / 1$, oestradiol $2200-3340 \mathrm{pmol} / \mathrm{l})$ or it may reflect a defect in the liver, affecting the production of SHBG.

The aetiology of PMS is still obscure but lower progesterone concentrations have been found in women with PMS, and Backström and Carstensen (1974) have shown high oestradiol in these women. This survey shows that women with severe PMS have a low SHBG binding capacity, and suggests that an increase in the free oestradiol at tissue level may cause an oestradiol/progesterone imbalance.

\section{Acknowledgments}

I wish to thank Dr Jawed Iqbal and Mr Stephen Virdee for their help in the assays; Dr Katharina Dalton for access to patients and Clin-Path Services Limited, London, for facilities, and Dr Jean Ginsberg for her help.

Dr Maureen E. Dalton was supported by grants from Ortho International Limited and Ciba Limited.

\section{References}

Dalton, K. (1977) The Premenstrual Syndrome and Progesterone Therapy. Wm Heinemann Medical Books, London.

Munday, M., Brush, M.G. \& Taylor, P.W. (1977) Progesterone and aldosterone levels in premenstrual tension. Journal of Endocrinological Proceedings, 73, 21.

BACKSTRÖM, T.L. \& CARSTENSEN, H. (1974) Oestrogen and progesterone in plasma in relation to premenstrual tension. Journal of Steroidal Biochemistry. 5, 257.

IQBAL, M.J. \& Johnson, M. (1977) A novel two-tier column technique to measure sex hormone binding globulin. Journal of Steroidal Biochemistry, 8, 977.

Backström T.L., Wide, L., Sodergard, R. \& Carstensen, H. (1976) FSH, LH, TeBG capacity, oestrogen and progesterone in women with premenstrual tension in the luteal phase. Journal of Steroid Biochemistry, 6, 473. 